\title{
Uma Análise do Processo de Planejamento de Trabalhos em Grupo no Ensino Superior
}

\author{
Edmar Welington Oliveira ${ }^{1}$, Marcos Roberto S. Borges ${ }^{2}$, Welington Veiga ${ }^{3}$ \\ ${ }^{1,3}$ Departamento de Ciência da Computação - Universidade Federal de Juiz de Fora \\ (UFJF) - Juiz de Fora, MG - Brasil \\ ${ }^{2}$ Departamento de Ciência da Computação - Universidade Federal do Rio de Janeiro \\ (UFRJ) - Rio de Janeiro, RJ - Brasil \\ \{edmar.oliveira, welington.veiga\}@ice.ufjf.br, mborges@nce.ufrj.br
}

\begin{abstract}
This research is focused on the difficulties inherent to the process of planning collaborative group work scenarios. It presents a study that shows some issues and inefficiencies associated with the process. The results show that educators are attempted to perform the design to their own practice - not compliant with pedagogical principles. Besides, they do not specify important learning parameters while planning such scenarios, indicating it is essential to provide them with proper and useful guidance.
\end{abstract}

Resumo. Este trabalho está focado nas dificuldades inerentes ao processo de planejamento de cenários colaborativos. Apresenta-se um estudo que expõe os problemas e ineficiências associadas ao processo. Os resultados mostram que educadores realizam o planejamento com base em suas próprias experiências, sem o suporte de modelos pedagógicos. Ademais, não especificam importantes parâmetros, indicando ser fundamental provê-los com adequada orientação.

\section{Introdução}

Processos instrucionais nem sempre são efetivos [Kimble et al., 2008]. Portanto, os objetivos inerentes à aquisição/desenvolvimento de habilidades e conhecimentos nem sempre são alcançados. Há um crescente interesse no uso da aprendizagem colaborativa (AC) de modo a se prover cenários que oportunizem aos alunos alcançar esses objetivos [Isotani et al., 2013]. Contudo, o simples fato de se ter estudantes trabalhando juntos não garante uma aprendizagem efetiva, ainda que recursos colaborativos lhe sejam providos [Weinberger et al., 2009]. Espera-se dos educadores a capacidade de criar cenários que mantenham alunos ativamente envolvidos na construção do próprio conhecimento; que, sobretudo, sejam adaptados a eles. A chance de se obter uma aprendizagem significativa e duradoura diminui sensivelmente quando não são apropriadamente planejados [Isotani et al., 2010]. De fato, planejamento inadequado é uma das principais razões de insucesso da aprendizagem em grupo [Dillenbourg, 2002; Strijbos et al., 2004].

Projetar cenários de AC é complexo, pois envolve inúmeros requisitos, variáveis e restrições [King, 2007]. Consequentemente, são indevidamente [Barkley et al., 2014] e ineficientemente estruturados [Höver \& Muhlhauser, 2014] - dificultando ou impedindo que alunos alcancem objetivos de aprendizagem. Trabalho em grupo, sobretudo quando o foco é aprendizado, requer planejamento cuidadoso [Dillenbourg, 2002]. Embora seja uma prática coletiva, é essencial ponderar sobre as necessidades individuais dos alunos. 
Soma-se à complexidade, transformar essas necessidades, e as intenções dos educadores, em parâmetros/variáveis que estruturem o trabalho. Esse é um processo particularmente desafiador para, mas não limitado a, educadores iniciantes - que, em geral, não possuem conhecimento e experiência necessários para planejar, adequadamente, cenários de AC [Isotani et al., 2013].

O problema de pesquisa refere-se à falta de planejamento adequado de trabalhos em grupo. Portanto, propôs-se analisar as dificuldades inerentes ao processo. O objetivo foi investigar e obter informações acerca da forma como docentes, do ensino superior da área de computação, realizam o planejamento de trabalhos em grupo ao lecionarem suas disciplinas na graduação presencial. Identificados os problemas/ineficiências, o enfoque de solução baseia-se na proposição de sugestões/ideias que os superem. A contribuição consiste em (a) expor e discutir os problemas identificados e (b) apresentar propostas de forma a resolvê-los ou minimizá-los. Nesse sentido, realizou-se um estudo exploratório com o propósito de obter informações quanto ao processo de planejamento realizado por docentes. O método qualitativo-quantitativo, aplicado ante uma amostragem intencional e não probabilística, foi utilizado. Os dados foram obtidos através de entrevistas. Análise de conteúdo e estatística foram empregadas para examiná-los.

O trabalho está organizado conforme a seguir. A seção 2 apresenta os resultados obtidos através das entrevistas. A seção 3 apresenta a análise dos dados e discute alguns dos problemas identificados. Ademais, apresenta propostas para solucioná-los, discorre sobre trabalhos relacionados ao contexto de planejamento de cenários de $\mathrm{AC}$ e apresenta uma proposta de trabalho futuro objetivando superar desafios, problemas e ineficiências das atuais abordagens voltadas a esse propósito. Na seção 4, conclusões e limitações da pesquisa são apresentadas.

\section{Planejamento de Trabalhos em Grupo: Um Panorama}

Para esta pesquisa, realizou-se um estudo exploratório. O objetivo foi investigar como professores, do ensino superior presencial em computação, planejam trabalhos em grupo ao ministrarem suas disciplinas de graduação. Foram entrevistados 30 indivíduos do departamento de ciência da computação de uma Universidade Federal. Optou-se pela realização de entrevistas semiestruturadas. Seguiu-se um roteiro de perguntas abertas, as quais foram previamente estabelecidas. Na presença do entrevistado, foram verbalmente realizadas em ordem previamente definida. $\mathrm{O}$ uso desse tipo de entrevista possibilitaria a realização de perguntas complementares, a depender das circunstâncias momentâneas da mesma, buscando clareza nas respostas e/ou a coleta de mais informações. Ademais, permitiria a posterior comparação de respostas. Em média, duraram 1 hora. Todas foram gravadas, com autorização. A amostra era formada por 27 doutores e 3 mestres. Quanto à experiência em docência no ensino superior, tinha-se: 6 com menos de 5 anos; 7 entre 5 e $10 ; 5$ entre 11 e 15; 8 entre 16 e $20 ; 2$ entre 21 e $30 ; 2$ com mais de 31 .

\subsection{Uso da Prática de Trabalho em Grupo}

Dos 30, 22 usam a prática de trabalho em grupo e 8, não. Para estes 8, perguntou-se se já a usaram; 5 , sim e 3, nunca. Para estes 3, perguntou-se o porquê de nunca terem usado; 1 disse preferir usar práticas individuais, por entender que o aluno aprende melhor. Já 2 disseram preferir aplicar exercícios, em sala, que podem ser feitos em dupla, se assim os alunos quiserem. Para àqueles 5 , questionou-se o porquê de não a utilizarem mais. As 
respostas, em geral, mencionaram dificuldade de planejamento dos trabalhos e problemas associados à cópias. Para esses 8 professores, a entrevista se encerrou após as perguntas acima. Entretanto, ela prosseguiu para os outros 22. Para estes, inicialmente, procurou-se saber (a) com que frequência fazem uso de trabalhos em grupo, (b) o que determina essa frequência e (c) por que fazem uso. Dos 22, 13 usam sempre que possível; 8, sempre e 1, quase sempre. Para 12, dos 13, a decisão depende das características da disciplina sendo ministrada: conteúdo, presencial ou $\mathrm{EaD}$, graduação ou pós-graduação, prática ou teórica, avançada ou básica, plano de curso livre ou fechado e quantidade de alunos matriculados. Foi mencionada ainda a experiência/preparação do professor para ministrar a disciplina. Quanto às razões de usarem, as respostas mencionaram: desenvolvimento de habilidades importantes ao mercado de trabalho, possibilidade de se propor tarefas mais complexas (demandando maior esforço do aluno), estimulo à interação e fomento à aprendizagem.

\subsection{Especificação Inicial do Trabalho}

Nenhum docente, ao projetar um trabalho, faz uso de modelos pedagógicos como suporte. Todos usam tão somente a própria experiência. Adotou-se a expressão "modelo pedagógico" como base comum às diferentes terminologias (p.ex., técnicas, pedagogias, teorias, padrões, etc.) empregadas no contexto de ensino/aprendizagem. Foi perguntado se é planejado um trabalho idêntico para toda a turma ou se são propostos trabalhos com características (complexidade, escopo, etc.) distintas, conforme necessidades particulares de aprendizado dos grupos e/ou alunos. Todos propõem um trabalho único para toda a turma, não analisando qualquer especificidade individual de alunos e/ou grupos.

\subsection{Formação dos Grupos}

Buscou-se saber se a formação dos grupos é deixada a cargo dos alunos ou se os professores a implementam. Todos disseram não interferir no processo. Questionou-se o porquê de não interferirem, que critérios eles imaginam que os alunos usam para formar os grupos e se isso poderia prejudicar o desenvolvimento do trabalho ou o aprendizado. Dos 22, 17 justificaram não interferir porque, como os alunos se conhecem, a formação dos grupos é mais fácil. Alunos poderiam formar grupos cujos integrantes possuem não apenas compatibilidade de horário para se reunirem, mas amizade. Assim, o grupo tende a ser mais "produtivo". Outros não se sentem à vontade para formar os grupos, pois não conhecem os perfis dos alunos. Logo, corre-se o risco de formar grupos cujos alunos não "se dão bem". Para outros, os alunos devem ter maturidade para formar grupos, pois no mercado de trabalho eles terão essa responsabilidade. Quanto aos critérios usados pelos alunos para a formação dos grupos, 21 disseram que o principal é a amizade. Dos 22, 13 não creem que deixar os alunos formarem os grupos prejudique o aprendizado. Segundo estes, o processo é favorecido justamente em função da amizade entre os integrantes do grupo. Para 9, o aprendizado pode ser comprometido - pois ao priorizarem a amizade, a composição do grupo nem sempre é apropriada. Ademais, por conta da amizade, muitos "acobertam" os que não fazem nada.

\subsection{Especificação das Atividades}

Quanto à especificação das atividades, buscou-se averiguar (a) o quão granular são definidas, se: (b) a descrição é explícita ou implícita, (c) a duração é estimada, (d) os objetivos de aprendizagem são especificados, (e) relações de dependência entre elas são definidas, (f) suas execuções são monitoradas, (g) o nível de conhecimento "adequado" 
para sua execução é estipulado e (h) como são divididas nos grupos. Por granularidade, considerou-se o nível de divisibilidade da atividade - se pode ser decomposta em tarefas mais específicas. Deste modo, a granularidade é proporcional à indivisibilidade. Nenhum dos professores especifica as atividades com alto nível de granularidade. Por descrição implícita, considerou-se aquela que está subentendida na descrição do trabalho. Por sua vez, a explícita refere-se àquela claramente expressada. Dos 22, 16 descrevem as tarefas explicitamente; 3 , implicitamente e 3 realizam ambas, a depender do trabalho. Quanto ao item "c", 21 não estimam a duração. Porém, 12 dos 21 o fazem para as etapas - definem um prazo para que um conjunto de atividades seja executado. Em relação ao item "d", 21 não definem objetivos de aprendizagem para tarefas. Quando o fazem, especificam para o trabalho como um todo ou para a disciplina. No item "e", considerou-se que relações de dependência entre atividades são aquelas estabelecidas de forma que a execução de uma não pode ser finalizada sem a conclusão de outra(s). Assim, 21 não definem esse tipo de vinculação.

Quanto ao item “f”, 16 não monitoraram a execução das atividades. Contudo, 6 o fazem. Para estes, o monitoramento está relacionado à definição das etapas, citadas no item "c". Neste item "c", 12 educadores disseram definir prazos para etapas do trabalho. Com a pergunta do item "f $\mathrm{f}$ ", verificou-se que 6 destes 12 educadores definem as etapas como uma estratégia de monitoramento. Nestas, os grupos se reúnem com o docente para discutir o progresso do trabalho e as atividades até então realizadas. Para os outros 6 , as etapas não são definidas para fins de monitoramento, mas apenas para entregas parciais do trabalho. Para o item "g", o nível de conhecimento adequado foi definido como sendo o intervalo entre o mínimo - exigido - e o máximo - permitido - para uma atividade ser realizada - de modo que a mesma não se apresente ao aluno como algo excessivamente complexo/inviável ou fácil/desestimulante. Nenhum docente especifica tarefas seguindo esse balizamento de conhecimento. Quando ponderado, o mínimo é definido em função dos pré-requisitos da disciplina ou de seu conteúdo. Para o item "h", 21 não interferem na divisão das tarefas. Para 17, o principal critério usado pelos alunos para a divisão é a habilidade/conhecimento individual do aluno. Assim, segundo os professores, os alunos se responsabilizam pelas atividades que lhe são mais fáceis.

\subsection{Avaliação da Aprendizagem}

Em relação à avaliação da aprendizagem dos alunos, procurou-se saber quais os critérios adotados e se é avaliado o grupo e/ou o aluno, individualmente. Os critérios se limitam à análise parcial/final do produto do trabalho (completude, correção, qualidade, etc.) e às apresentações orais realizadas pelos alunos. Dos 22, 3 avaliam apenas o produto do trabalho; 19 avaliam apresentações e produto - destes, 6 avaliam as entregas parciais realizadas durante o desenvolvimento do trabalho. Dos 22, 18 avaliam o grupo e alunos; 2 , somente o grupo e 2, apenas o aluno. Para os 20 que avaliam o aluno, foi questionado se realizam uma análise a priori dos mesmos para averiguar o aprendizado ao término do processo/trabalho. Destes 20, 14 não realizam qualquer análise; 6 disseram fazê-la, mas informalmente (observando desempenho em disciplinas anteriores, avaliações da própria disciplina, etc.). Quanto à atribuição de nota, 17 definem uma mesma nota para todos os membros do grupo. Os outros 5 atribuem notas individuais. Foi perguntado se entregar o trabalho bem desenvolvido é garantia de aprendizado. Para 18, não - pois alguns alunos podem não participar do seu desenvolvimento. A possibilidade de o mesmo ser fruto de cópia também foi mencionada. Igualmente, foi perguntado se entregar um trabalho mal 
desenvolvido significa que não houve aprendizado. Para 20, pode ocorrer aprendizagem decorrente do processo de desenvolvimento. Questionados sobre o(s) motivo(s) de alguns grupos falharem ao realizar um trabalho, 15 citaram falta de organização/gerenciamento do grupo quanto ao processo de desenvolvimento do trabalho como principal motivo. Falta de motivação e interesse dos alunos, falta de interação e formação inapropriada dos grupos também foram citadas. Perguntados sobre quem deveria ser responsabilizado nos casos de falha, 9 disseram os alunos; 2, os docentes e 11, ambos. Os professores por não projetarem adequadamente o trabalho e não monitorarem seu desenvolvimento; os alunos pela desorganização em relação à sua realização.

\subsection{Trabalho em Grupo e Colaboração}

Foi perguntado se os alunos sabem trabalhar em grupo de forma efetiva. Para 13, não; 2, sim; 7 afirmaram que depende do aluno. Para os 13, alunos não sabem organizar, gerenciar o desenvolvimento do trabalho - incluindo como interagir, dividir as tarefas e responsabilidades de maneira igualitária entre membros do grupo, etc. Alguns disseram que nem todos os alunos possuem maturidade suficiente para esse tipo de prática, e que outros são resistentes a qualquer modelo de ensino que se afaste do tradicional (centrado no professor e explanatório). Para outros, os próprios docentes são responsáveis por essa inaptidão dos alunos, pois não encorajam trabalhos em grupo; e quando o fazem, não os planejam adequadamente. Para os 7, depende do interesse/motivação dos alunos. Quando perguntados sobre qual seria a principal motivação dos alunos em participar de práticas em grupo, todos responderam a realização do trabalho e não o aprendizado. Embora haja exceções, estão mais interessados em desenvolver o trabalho para cumprir os requisitos da disciplina e a consequente aprovação - e todas as suas ações, apropriadas ou não, são direcionadas neste sentido.

Com relação à colaboração, foi perguntado se ela é importante para se promover a aprendizagem. Para 21, sim. Para 1, ela não é benéfica em toda situação, pois há casos em que o aluno deve desenvolver certas características (criatividade, pensamento lógico, etc.) por conta própria. Perguntados se todo trabalho é colaborativo, 21 responderam não. Para estes, por vezes, alunos apenas dividem tarefas entre si e trabalham individualmente - sem interação. Em outras, um membro do grupo (ou poucos) realiza todo o trabalho (ou grande parte). Foi perguntado se os alunos possuem consciência do que é colaborar. Para 15, não; 3, sim; para 4, depende do aluno (alunos em final de curso têm essa consciência, ao contrário daqueles em início de curso). Para os 3, os alunos a tem, mas optam por não usar o conceito por estarem mais interessados em realizar o trabalho. Perguntados se, ao planejarem os trabalhos, definem instruções/diretrizes que auxiliem/orientem os alunos a trabalharem juntos (colaborarem), todos alegaram não definir nada neste sentido.

\subsection{Planejamento Detalhado}

Buscou-se verificar se, na opinião dos docentes, realizar um planejamento mais detalhado pode fomentar um aprendizado mais efetivo. Por detalhado, considerou-se os parâmetros evidenciados na entrevista. Dos 22, 15 disseram sim; 5, não e 2 responderam que depende do nível de detalhe. Para estes últimos 5, realizar um planejamento "muito detalhado" pode "minar" a criatividade e a flexibilidade desejáveis em um processo de aprendizagem. Para aqueles 15, maior detalhamento é benéfico por possibilitar orientar as ações dos alunos, incentivar a interação e facilitar o monitoramento e a avaliação. Ao serem perguntados se efetuar um planejamento detalhado é complexo, 15 disseram sim 
- uma vez que há muitas variáveis a considerar e pelo fato de a maioria dos professores não possuir conhecimentos sobre teorias/abordagens pedagógicas que os auxiliariam no processo. Para 7, não há complexidade. Assim, nos casos em que docentes não realizam um planejamento detalhado, a justificativa está no fato de ser uma "opção" por acharem o processo muito "trabalhoso". Solicitados a classificar o planejamento que realizam, 3 o classificaram como detalhado; 7, não detalhado; 12, "médio". Buscou-se averiguar se seria benéfico ao professor dispor de uma infraestrutura (metodologia, ferramenta, guia, etc.) que o auxiliasse a planejar seus trabalhos. Dos 22, 20 responderam sim. Para estes, seria útil uma infraestrutura que os auxiliassem no monitoramento, definição de objetivos de aprendizagem, divisão de grupos, análise do aprendizado, etc.

\section{Análise dos Resultados}

As entrevistas salientaram vários problemas e ineficiências quanto ao processo de planejamento realizado pelos professores - principalmente com relação à orientação das ações dos alunos e a analise do aprendizado. Para a maioria dos docentes, os estudantes não sabem trabalhar em grupo de forma efetiva, não têm consciência do que é colaborar e estão, prioritariamente, empenhados tão somente na realização/entrega do trabalho. As consequências, diretas ou indiretas, englobam interações ineficazes/inexistentes, divisão indevida de tarefas/responsabilidades, má organização no desenvolvimento do trabalho e condutas desfavoráveis à aprendizagem (p.ex., aluno executar somente tarefas que lhe são mais "fáceis"). Além disso, a maioria crê que as falhas na realização do trabalho são decorrentes de más ações dos alunos - e quase a metade afirma que a responsabilidade é exclusiva dos mesmos. Apesar do exposto, o planejamento não é realizado de maneira a orientar os alunos. De fato, os professores não orientam a formação de grupos, a divisão de tarefas e responsabilidades, não definem diretrizes que fomentem a interação entre os alunos, não especificam detalhadamente as atividades (duração, relação de dependência, etc.) e não monitoram a execução das mesmas. É essencial que o trabalho seja planejado de maneira a orientar o aluno quanto à sua conduta, às atividades que deve executar e às interações com seus pares. Essa orientação faz-se necessária, pois os alunos (por vezes) não realizam atividades colaborativas adequadamente [Alharbi et al., 2014.]. Além disso, a "colaboração livre" (não estruturada) nem sempre produz resultados de aprendizagem satisfatórios [Alharbi et al., 2014; Dillenbourg, 2002; Barkley et al., 2014].

Quanto ao acompanhamento e análise do aprendizado, averiguou-se que não são especificados parâmetros que possibilitem ao docente realizá-los de forma precisa/exata - principalmente com relação ao aluno, individualmente. A maioria dos professores não realiza uma análise a priori dos estudantes (no que tange às suas necessidades/interesses de aprendizado, características, etc.). Além disso, o trabalho e as tarefas que o compõem não são estruturados de forma a atender às especificidades dos mesmos. Muitos utilizam o produto do trabalho para avaliar o aprendizado, mesmo não considerando que entregar o mesmo por si só (ainda que bem desenvolvido) seja garantia da aprendizagem. Outros, embora adotem apresentações, não especificam critérios, baseando-se no "feeling" ao se observar o aluno (sua capacidade de se expressar, responder, demonstrar conhecimento, etc.) durante as mesmas. Embora a maioria afirme avaliar os alunos individualmente, as notas não são individuais. Dado o exposto, somado ao fato de muitos não monitorarem o desenvolvimento do trabalho e/ou o processo de aprendizagem, é discutível a precisão e justeza dos professores quanto à percepção do aprendizado dos alunos. Em termos da AC, dois itens devem ser avaliados: o conhecimento do aluno quanto ao conteúdo e sua 
VI Congresso Brasileiro de Informática na Educação (CBIE 2017)

Anais do XXVIII Simpósio Brasileiro de Informática na Educação (SBIE 2017)

participação nos processos do grupo [Barkley et al., 2014]. Contudo, avaliar e monitorar o progresso individual em trabalhos em grupo é uma tarefa desafiadora [Fidalgo-Blanco et al., 2015] - justificando a proposição de infraestruturas/metodologias que orientem os educadores.

\subsection{Propostas de Respostas aos Problemas Identificados}

Um planejamento cuidadoso é essencial para a efetividade do processo de ensino e aprendizagem [Dillenbourg, 2002]. Entretanto, como evidenciado na seção anterior, os educadores não apenas deixam de definir vários parâmetros importantes, como aplicam práticas e/ou adotam condutas/posturas, por vezes, inadequadas e/ou ineficientes. Vários problemas intrínsecos à prática de trabalhos em grupo podem ser amenizados ou mesmo resolvidos a partir de decisões de planejamento. Uma delas refere-se à granularidade das atividades. Propõe-se que sejam especificadas com alta granularidade - tarefas menores. Argumenta-se que isso não apenas facilitaria a definição dos seus parâmetros (objetivos de aprendizagem, duração, etc.), como possibilitaria realizar, de maneira mais precisa, a avaliação e o monitoramento do processo de aprendizagem. Ainda, auxiliaria na divisão das mesmas nos grupos e na organização do processo de desenvolvimento pelos alunos. De modo a fomentar a interação/colaboração entre alunos, propõe-se a especificação de relações de dependência entre atividades. De fato, a interdependência entre tarefas é um importante aspecto do trabalho colaborativo [Raposo et al., 2001]. Quando dependências entre tarefas não são definidas, oportuniza-se que alunos as realizem sem que interações entre eles ocorram - logo, trabalho individual dentro de um contexto coletivo.

Como mencionado, projetar cenários de AC é complexo, pois envolve inúmeros requisitos, variáveis e restrições. As entrevistas evidenciaram que (todos) os professores planejam cenários de trabalho em grupo a partir de suas próprias experiências. Modelos pedagógicos, portanto, não são utilizados como suporte ao processo. Consequentemente, vários parâmetros importantes não são definidos. Alguns educadores sequer tem ciência dos mesmos. Sem o suporte destes modelos, o docente pode aplicar/replicar práticas, por vezes, ineficientes, no que tange à aprendizagem. Além disso, problemas decorrentes de um inadequado planejamento podem desestimular alunos e professor (desencorajando-o com relação à metodologia). Dos professores que alegaram não usar trabalho em grupo, muitos não o fazem em virtude de más experiências (em parte, consequências diretas de um mau planejamento). Portanto, o uso de modelos pedagógicos para auxiliar o processo torna-se importante. Projetar cenários colaborativos sem considerações pedagógicas não necessariamente melhora os resultados de aprendizagem e pode, inclusive, prejudicar o desenvolvimento dos alunos [Dillenbourg \& Hong, 2008].

\subsection{Abordagens para o Planejamento de Trabalhos em Grupo}

No contexto de planejamento de cenários de $\mathrm{AC}$, uma tendência de pesquisa tem sido scripts colaborativos (SC). Um SC é um conjunto de instruções/diretrizes que possibilita estruturar e descrever cenários de AC, através da definição de componentes (atividades, alunos, recursos, etc.) e mecanismos (distribuição e sequenciamento de tarefas, formação de grupos, etc.) [Kobbe et al., 2007]. Seu objetivo é estimular e guiar a colaboração entre alunos [Dillenbourg, 2002]. Dada a complexidade intrínseca à sua estruturação, muitas ferramentas de autoria têm sido propostas para auxiliar educadores no processo [Challco et al., 2016; Alharbi et al., 2014; Fernández et al., 2013]. Embora algumas implementem modelos pedagógicos como modo de prover suporte educacional ao desenvolvimento de 
SC, a quantidade de modelos suportados é limitada. Essa restrição, em parte, decorre da complexidade intrínseca a alguns modelos, em termos da sua compreensão [Isotani et al., 2010], além de dificuldades técnicas e dos custos relacionados à sua implementação nas ferramentas [Fragoso et al., 2014].

Usualmente, a representação de scripts é realizada usando-se uma linguagem de modelagem educacional (EML) [Challco et al., 2016] - sendo IMS-LD a mais utilizada. $\mathrm{O}$ intuito é prover interoperabilidade, possibilitando que os scripts sejam executados em sistemas de gerenciamento de aprendizagem (LMS) compatíveis com a mesma. Apesar do suporte oferecido por tais ferramentas, o processo de planejamento é restrito aos seus recursos pedagógicos/técnicos; uma potencial limitação, dado o desafio das mesmas em conseguir acompanhar as constantes inovações do domínio pedagógico. Uma alternativa ao desenvolvimento destes recursos pelos mantenedores de ferramentas é a utilização de "recursos terceirizados" - criados por diferentes organizações/instituições. Em [Lorenzo et al., 2008], essa abordagem é considerada. Contudo, trata-se de um LMS (e não de uma ferramenta de autoria) - portanto, uma estrutura não voltada à concepção de scripts, mas somente à sua execução. Além disso, scripts criados especificamente para esse LMS, de modo a usar recursos terceirizados por ele providos, só podem ser executados no mesmo - um prejuízo à interoperabilidade. Algumas ferramentas de autoria, LAMS [Kordaki et al., 2012], IWT [Capuano et al., 2013], etc., se propõem não apenas à criação dos scripts, mas à sua execução. Contudo, o processo é igualmente limitado aos recursos oferecidos pelas mesmas.

\subsection{Uma Proposta para Auxiliar o Planejamento de Trabalhos em Grupo}

Os resultados obtidos com as entrevistas indicam a necessidade de se prover aos educadores infraestruturas que os auxiliem no processo de planejamento de trabalhos em grupo. Embora existam ferramentas de autoria voltadas a este propósito, todas possuem limitações [Alharbi et al., 2014; Challco et al., 2016], e muitas não proveem suporte para especificação de vários parâmetros relevantes ao processo de aprendizagem [Fernández et al., 2013]. As restrições presentes nas atuais abordagens, e os problemas/ineficiências observados no estudo exploratório (seção 2), fomentaram a idealização de uma proposta (trabalho futuro da presente pesquisa) para assistir o planejamento de trabalhos em grupo. Tal proposta consiste em viabilizar a integração de recursos educacionais em ambientes virtuais de aprendizagem (AVA) como diretriz para planejamento de trabalhos em grupo. A integração é direcionada no sentido de se possibilitar incorporar/adicionar soluções de terceiros - uma propriedade indispensável (pelo exposto na seção 3.2). Nesse sentido, o uso de serviços web torna-se estratégico, pois estruturar recursos educacionais na forma de serviços permite que seu desenvolvimento e evolução ocorram alheios às plataformas tecnológicas e que eventuais correções se tornem disponíveis instantaneamente. Porém, é quase inviável que alguma plataforma consiga incorporar todas as inovações/soluções, em virtude das especificidades/fragmentação das mesmas [Fragoso et al., 2014]. Portanto, uma alternativa é a perspectiva de ecossistemas de software (ECO) [Santos, 2011], pois possibilita que diferentes organizações e/ou instituições contribuam com soluções para a estruturação de ambientes de aprendizagem ricos em diversidade de serviços.

Formalmente, um ECO é “... uma interação de um conjunto de atores sobre uma plataforma tecnológica comum, que resulta em um aglomerado de serviços de software. Cada ator é motivado por seus interesses, conectando-se aos demais e ao ECO como um todo através de relacionamentos simbióticos. A plataforma tecnológica é estruturada de 
VI Congresso Brasileiro de Informática na Educação (CBIE 2017)

Anais do XXVIII Simpósio Brasileiro de Informática na Educação (SBIE 2017)

maneira a possibilitar o envolvimento/contribuição dos diferentes atores..." [Manikas \& Hansen, 2013]. A proposta, portanto, visa transformar AVA em plataformas/ambientes que sustentem um ecossistema - assim, permitindo que serviços voltados ao processo de planejamento (providos por quaisquer desenvolvedores externos) sejam integrados. Essa estratégia, neste sentido, dispensa plataformas de implementá-los - logo, uma vantagem sobre as atuais abordagens. Ademais, viabiliza não apenas a autoria, mas a execução dos cenários de aprendizagem. Diferentemente de [Lorenzo et al., 2008], os serviços externos estão "atrelados" ao ecossistema (que pode ser implementado em qualquer LMS); e não à plataforma LMS. Portanto, não se compromete a interoperabilidade.

\section{Conclusões}

O planejamento de cenários de aprendizagem colaborativa é complexo - motivo pelo qual são indevidamente e ineficientemente estruturados, dificultando ou impedindo que alunos alcancem objetivos de aprendizagem. Neste artigo, buscou-se analisar como educadores, do ensino superior presencial em computação, planejam trabalhos em grupo ao lecionarem suas disciplinas de graduação. Realizou-se entrevistas com 30 educadores de uma Universidade Federal. Os resultados evidenciaram que eles realizam o processo de planejamento com base apenas em suas próprias experiências. Ademais, não definem parâmetros essenciais - principalmente com relação à orientação das ações dos alunos e analise do seu aprendizado. As dificuldades dos educadores para com o processo indica a necessidade de prover-lhes apropriada orientação, expondo-os a parâmetros que devem ser considerados no processo.

Quanto às limitações da pesquisa, aponta-se a não representatividade da amostra examinada. Entretanto, presume-se que os problemas identificados sejam intrínsecos ao contexto de trabalho em grupo e, portanto, verificados (mesmo que em parte) em outras amostras. É objetivo da pesquisa estender a análise para docentes de outras instituições (a princípio, mantendo-a no domínio da computação). Trabalhos futuros compreendem, além da extensão da análise, o desenvolvimento da proposta discutida na seção 3.3 - de modo a auxiliar educadores no planejamento de cenários de $\mathrm{AC}$ e que supere problemas e ineficiências das atuais abordagens voltadas ao mesmo propósito.

\section{Referências}

Alharbi, N. M., Athauda, R. I., Chiong, R. (2014) A Survey of CSCL Script Tools That Suport Designing Collaborative Scenarios. Web and Open Access to Learning.

Barkley, E. F., Major, C. H., K., Cross, K. P. (2014). Collaborative Learning Techniques: A Handbook for College Faculty. San Francisco: Jossey-Bass.

Challco, G. C., Bittencourt, I. Ig., Isotani, S. (2016). Computer-based systems for automating instructional design of collaborative learning scenarios: a systematic literature review. International Journal of Knowledge and Learning, 11(4).

Capuano, N., Miranda, S., Ritrovato, P., Mangione, G.R. and Pierri, A. (2013). Design and execution of dynamic collaborative learning experiences, International Journal of e-Collaboration, 9(1), 26-41.

Dillenbourg, P., Hong, F. (2008). The mechanics of CSCL macro scripts. International Journal of Computer-Supported Collaborative Learning, 3(1), 5-23. 
VI Congresso Brasileiro de Informática na Educação (CBIE 2017)

Anais do XXVIII Simpósio Brasileiro de Informática na Educação (SBIE 2017)

Dillenbourg, P. (2002). Overscripting CSCL the risks of blending collaborative learning with instructional design. Three worlds of CSCL. Can we support CSCL? 61-91.

Fidalgo-Blanco, A., Sein-Echaluce, M. S., García-Peñalvo, F. J., Conde, M. A. (2015) Using Learning Analytics to improve teamwork assessment, C.H.B, 47, 149-156.

Fernández, E. V., Leo, D. H, Pérez, J. I. A., Dimitriadis, Y. (2013). WebCollage: an implementation of support for assessment design in CSCL macro-scripts, Computers \& Education, 67, 79-97.

Fragoso, O. G., Santaolaya, R., Munoz, S. J., Valenzuela, B. D., Rojas, J. C. (2014). Integration of learning Web services into LMS. America/Panama Convention, 1-6.

Höver, K. M.; Mühlhäuser, M. (2014). Can We Use S-BPM for Modeling Collaboration Scripts?. Communications in Computer and Information Science. 174-187.

Isotani, S., Mizoguchi, R., Isotani, S., Capeli, O. M., Isotani, N., Albuquerque, A. R., Jaques, P. (2013). A Semantic Web-based authoring tool to facilitate the planning of CL scenarios compliant with learning theories. Computers/Education, 63, 267-284.

Isotani, S., Mizoguchi, R., Inaba, A., \& Ikeda, M. (2010). The foundations of a theoryaware authoring tool for CSCL design. Computers \& Education, 54(4), 809-834.

Kimble, C., Hildreth, P. M., \& Bourdon, I. (2008). Communities of practice: Creating learning environments for educators, Vol. 1. Charlotte, NC: Information Age Pub.

King, A. (2007). Scripting Collaborative Learning Processes: A Cognitive Perspective. Scripting Computer-Supported Collaborative Learning, 13-37.

Kobbe, L., Weinberger, A., Dillenbourg, P., Harrer, A., Hamalainen, R., F, F. (2007). Specifying Computer-Supported Collaboration Scripts. Journal CSCL, 2, 211-224.

Kordaki, M., Daradoumis, T., Fragidakis, D. and Grigoriadou, M. (2012). Adapting the collaborative strategy students team achievement divisions in an information technology work place. Springer. Studies in Computational Intelligence, 131-153.

Lorenzo, B. M. L., Sánchez, E. G., Gorgojo, G. V., Dimitriadis, Y. A., Pérez, J. I. A, Abellán, I. M. (2008). Gridcole: A tailorable grid service based system that supports scripted collaborative learning, Computers \& Education, 51, 155-172.

Manikas, K., Hansen, K. (2013) Software Ecosystems: A Systematic Literature Review. Journal of Systems and Software, 86(5), 1294-1306.

Raposo, A. B., Magalhães, L. P., Ricarte, I. L. M., Fucks, H. (2001). Coordination of Collaborative Activities A Framework for the Definition of Tasks Interdependencies. Workshop on Groupware, Darmstadt, Germany, 170-179.

Santos, R; Engenharia e Gerenciamento de Ecossistemas de Software (2013). Tese Doutorado. Pós-graduação em Engenharia de Sistemas e Computação, UFRJ.

Strijbos, J. W., Martens, R. L., \& Jochems, W. M. G. (2004). Designing group based learning: six steps to designing computer-supported group based learning. Computers \& Education, 42, 403-424.

Weinberger, A., Kollar, I., Dimitriadis, Y., Mäkitalo-Siegl, K., \& Fischer, F. (2009). Computer-supported collaboration scripts. Educational psychology and CS, 155-173 